\title{
Subspace Feature Analysis of Local Manifold Learning for Hyperspectral Remote Sensing Images Classification
}

\author{
Ling Ding ${ }^{1,2, *}$, Ping Tang ${ }^{1, *}$ and Hongyi $\mathrm{Li}^{1}$ \\ ${ }^{1}$ Institute of Remote Sensing and Digital Earth, Chinese Academy of Sciences, Beijing, China \\ ${ }^{2}$ University of Chinese Academy of Science, Beijing 100039, China
}

Received: 26 Aug. 2013, Revised: 28 Nov. 2013, Accepted: 29 Nov. 2013

Published online: 1 Jul. 2014

\begin{abstract}
Dimensionality reduction and segmentation have been used as methods to reduce the complexity of the representation of hyperspectral remote sensing images. In this study, a new object-oriented mapping approach is proposed based on nonlinear subspace feature analysis of hyperspectral remote sensing images. Nonlinear local manifold learning approaches for feature extraction were utilized to obtain subspace feature representation of hyperspectral remote sensing images. Afterwards, with a proper selection of parameters, the extracted subspace feature images were fed into the object-oriented system. Texture features derived from gray level co-occurrence matrix and wavelet filter with the use of SVM classifier at the pixel level of the feature images were also used to evaluate the proposed algorithm. Experiments are conducted on the AVIRIS dataset with 220 spectral bands, covering an agricultural area. Classification results show that the proposed object-oriented subspace analysis approach can give significantly higher accuracies than the traditional pixel-level and texture-based subspace feature classification.
\end{abstract}

Keywords: Nonlinear manifold learning, subspace feature analysis, object-oriented classification, hyperspectral remote sensing images, texture

\section{Introduction}

Hyperspectral sensors are characterized by the high spectral resolution that provides a very large number of wavelength channels per pixel [1]. These narrow and contiguous spectral channels improve the capabilities for discriminating, characterizing and monitoring over an area. Theoretically, hyperspectral data should increase abilities in classifying land use/cover types. However, the "curse of dimensionality" and highly correlated spectral bands are problematic for many operations on hyperspectral data such as model fitting, parameter estimation or optimization of objective functions, the problem of classification is often referred to the "Hughes phenomenon" [2-4]. Hyperspectral data also allow better characterization of nonlinear responses, which is not possible with multispectral data. The samples of hyperspectral data often lie on a nonlinear subspace of lower dimension. There are multiple sources of nonlinearity, one of the more significant sources, especially in land-cover classification application, stems from wavelength dependent nonlinear reflectance defined by the bidirectional reflectance distribution function (BRDF). Another source of nonlinearity arises from complex scattering of energy in vegetation and nonlinear attenuation of energy in water bodies [5].

Dimension reduction (DR) has been used to mitigate the issues of high interband spectral correlation and the "Hughes phenomenon" for classification of hyperspectral data. The resultant features not only require reduced data storage and computation, but also produce a more robust (small variance) and accurate (small bias) classifier. Several unsupervised and supervised linear feature extraction methods have been applied to hyperspectral data such as principal component (PCA) [6], maximum noise fraction (MNF) [7], nonparametric weighted feature extraction (NWFE) [8]. Recently, the impact of feature selection has been also investigated with respect to support vector machines (SVM) which do not necessarily require a separate feature selection/extraction step [9]. the SVM classifier is well known for its robustness to high dimensionality, removal of irrelevant features can still improve the performance of SVM classifier for some hyperspectral data, but these linear feature extraction and

\footnotetext{
*Corresponding author e-mail: xiaodingdj@126.com, tangping@irsa.ac.cn
} 
classification methods largely ignore the inherent nonlinear characteristics and cannot properly handle the nonlinearity in hyperspectral data, often resulting in extraction of non-representative features and low classification accuracies. Recently, the machine learning community has demonstrated the potential of manifold learning (ML) methods for nonlinear DR; nonlinear ML algorithms assume that the original high dimensional data actually lie on a low dimensional manifold defined by local geometric differences between samples. Recent research has demonstrated the potential of ML approaches for the representation of high dimensional observations through nonlinear mapping [10].

Nonlinear ML methods are broadly characterized as global or local approaches. Global ML methods retain the fidelity of the overall topology of the data set such as isometric feature mapping (Isomap) [11] and kernel PCA(KPCA) [12], but have greater computational overhead for large data sets, while local ML methods preserve local geometry Isometric feature and are computationally efficient because they only require sparse matrix computations such as locally linear embedding (LLE) [13], local tangent space alignment (LTSA) [14], and Laplacian eigenmaps(LE) [15]. These methods are developed to represent high dimensional nonlinear phenomena in lower dimensional spaces, the imbedded features have been used to address the nonlinearity in hyperspectral data; they are potentially useful for classification of hyperspectral data. Bachmann et al [5]demonstrated Isomap potential for data representation and classification of hyperspectral data. The problem of heavy computational load for large-scale remote sensing data sets was tackled by a scalable approach based on various methods for aligning manifolds derived from image subsets, experiments showed that features extracted by Isomap were able to explain a greater amount of variance in the data than MNF coordinates; The proposed ENH-Isomap was used for bathmetric retreival, and produced retrieval errors for spatially and temporally disjoint test sets that were comparable to those of bathymetric LiDAR [10]. Fauvel et al [16]applied kernel PCA (KPCA) to airborne hyperspectral data sets prior to the application of support vector machines (SVM), and showed that KPCA extracts more informative features than conventional PCA, justifying the use of KPCA in combination with SVM-based classification. Li et al [17] proposed a new ML technique called discriminant locally linear embedding (DLLE), in order to preserve the local geometric properties within each class and enhance the separability between different classes. Ma et al [18]generalized a new local $\mathrm{ML}$ weighted $\mathrm{kNN}$ classifier from the kernel view and applied it to hyperspectral images classification,the study showed that the proposed method outperforms the regular k-NN method in classification of hyperspectral data.

By summarizing nonlinear ML methods and classification methods for hyperspectral data, it can find that these approaches always focus on pixel-wise classification which only utilizes spectral information associated with a given pixel location and ignores important spatial information. This important spatial information is recognized as characteristics of geographical data, including region shape, location and relation of neighboring samples to the targeted sample. Pixel-based classification often leads to a "pepper-and-salt" appearance it is also difficult to flag outliers in a homogeneous area and discriminate the spectrally similar class when contextual information is not considered. Few such approaches for the exploitation of spatial information is necessary for classification of hyperspectral imagery have been proposed, which is partly due to the high dimensionality of the data and the spectral and spatial heterogeneity of remote sensing images $[19,20]$.

In this study, for the sake of exploiting both spectral and spatial information contained in hyperspectral remote sensing images, an object-oriented nonlinear subspace feature analysis (OSFA) for hyperspectral remote sensing images classification was proposed. Local ML such as LLE, LE and LTSA methods were employed to reduce the number of the dimensionality and extract the spectral feature subspace from hyperspectral remote sensing images. The proposed approach operated on many more object-related features than typically available with pixel-based approaches. The idea of object-based analysis is to group the neighbouring pixels into spectrally homogeneous objects.Multiresolution segmentation in Definiens? Professional 7.0 (DEFINIENS 2007) [21] was utilized to extract objects from feature images and SVM classifier was then used to classify the object-based feature images. In addition, GLCM and wavelet-based texture features were implemented at the pixel of subspace feature images and the classification results were used to evaluate the proposed algorithm.

\section{Methods}

\subsection{Local manifold learning}

LLE, LE and LTSA were selected for experiment analysis, they are based on solely preserving the properties of small neighbourhoods around the data points. They are initiated by constructing a nearest neighbourhood graph for each data point, and the local structures are then used to obtain a global manifold. According to the framework by solving the eigenvalue problem $L Y=\lambda B Y$, the embedding $Y$ of target dimension $p$ is provided by the eigenvectors corresponding to the $2 \sim p+1$ smallest eigenvalues(the eigenvector that corresponds to the smallest zero eigenvalue is a unit vector with equal elements and is discarded).The parameters and computational complexity are listed in Table 1. $N$ is the number of data sample. $D$ is 
Table 1: The parameters and computational complexity of local ML

\begin{tabular}{ccc}
\hline Method & Parameter & Complexity \\
\hline LLE & $p, k$ & $O\left(D N^{2}\right)$ \\
LTSA & $p, k$ & $O\left(D N^{2}\right)$ \\
LE & $p, k$ & $O\left(D N^{2}\right)$ \\
\hline
\end{tabular}

dimension of the original space of data samples. Another possibility is to connect every point to all other points within a given radius $\varepsilon$, We did no use this type of graphs since the $k$-nearest-neighbours graph yielded good results.

\subsection{Object-oriented nonlinear subspace analysis}

The object-oriented classification based on objects is a new classification method, it allows explicit consideration of spatial context during the classification processing. An image is subdivided into separated regions called objects or segments during the segmentation process, and then the objects are assigned to a specific class. Object-oriented classification has been applied successfully to high resolution remote sensing image the high resolution satellite images have richer spatial but less spectral information, such as Quickbird, IKONOS, and GeoEye only contain four spectral bands [22, 23]. Increased number of spectral bands in hyperspectral data provides abundant information on earth surface by capturing the signature of a ground object in narrow spectral bands over contiguous spectral ranges. Not only it helps differentiating ground objects through characteristic wavelengths, but also allows investigation on more complex natural phenomena that are often nonlinearly related to the spectral information. Linear dimension reduction methods may fail to precisely relate the spectral changes to the nonlinear factors. Therefore, linear feature extraction and object-oriented analysis cannot be directly applied to hyperspectral data. In this study, nonlinear ML methods and the object-oriented classification technique were integrated in order to make use of both spectral information (feature vector of the pixels) and spatial information (size, shape and adjacency to other pixels) for hyperspectral data. The proposed OSFA approach involves main steps as follows:

Step 1: Preprocessing: The low-dimensional feature images are extracted from the originally high dimensional hyperspectral remote sensing images via local ML methods. It reduces redundant hyperspectral information, thus raising the efficiency of the data processing. In this study, Because of memory and computational constraints in local ML. the hyperspectral remote sensing images are divided into a set of $50 \times 50$ pixel tiles. Local ML methods focus inherently on retaining local structure of the data, so, manifold coordinates of each tile can be thought to lie on a unified full-scene global manifold coordinate system.

Step 2: Multiresolution segmentation algorithm: the subspace feature images are segmented using the multi-resolution segmentation algorithm (Definiens 2007), which starts with one-pixel image segments and merges neighbouring segments together until a 'heterogeneity threshold' is reached. The heterogeneity threshold is determined by a user-defined scale parameter, as well as smoothness/compactness weights $[24,25,26]$. In this study, Different scales, from 5 to 50, are applied to each pair of weights for segmenting the feature images. Since this study is on land use classification and does not emphasize any kind of class to be extracted, so the weight of each image layer (band) is the same and takes default value. Appropriate segmentation parameters are determined subjectively by comparing segmentation results with class label maps. Classification accuracies are also used for comparing segmentation alternatives and deciding optimal segment size.

Step 3: Spectral characteristics of objects: After Step (2), the feature images have been represented based on objects. Accordingly, the pixel-by-pixel spectral information within each object is integrated for object-based features. In this paper, the spectral characteristic for each object is calculated by averaging the spectral vectors of all pixels within this object:

$$
F_{b}(i)=\frac{1}{n} \sum_{x \in i} F_{b}(x)
$$

with $F(x)=\left\{F_{1}(x), \cdots, F_{b}(x), \cdots, F_{D}(x)\right\}$. Where $F(x)$ is the spectral vector with $D$-dimensional space for pixel $x$. After this step, each segment $i$ is represented using an averaged spectral vector with dimension of $D$.

Step 4: SVM-based classification: Each object is assigned to a specific class using SVM classifier. The radial basis function (RBF) kernel is used due to its effectiveness in many classification problems. The regularization parameter and the spread factor of RBF kernel are determined using cross-validation approach.

Step 5: Accuracy assessment: Classification accuracy is measured for both classification methods using a standard error matrix. An accuracy assessment of the classification results is performed. The accuracy assessment is implemented on the basis of classification results and reports overall accuracy. 


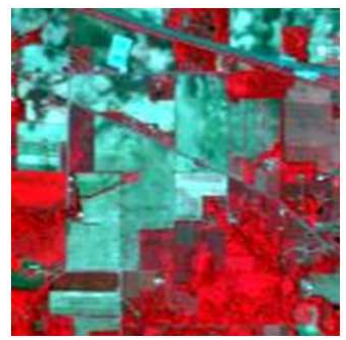

(a)

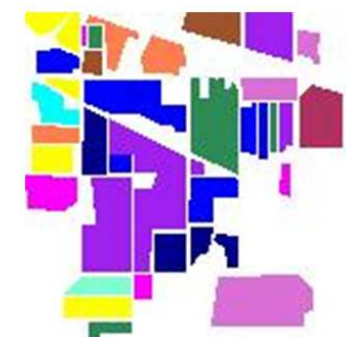

(b)

Fig. 1: RGB image (channels 50, 26, 16 for RGB) (a) and the ground-truth reference map: class label (Number of training samples/number of test samples) for the AVIRIS data set (b)

\section{Results and discussions}

\subsection{AVIRIS dataset}

A sub-image of AVIRIS data which was obtained from ftp://ftp.ecn.purdue.edu/biehl/MultiSpec/ with the size of $145 \times 145$ pixels was used. This experiment included the Indiana Indian Pine with the 220 spectral channels and 12-class data set acquired by the AVIRIS sensor in June 1992 at 20-m spatial resolution. After removed water absorption bands (104-108,150-163, and 220), 200 bands were available for analysis. The scene showed an agricultural region with regular geometry. Selected results were included for $\mathrm{C} 2$ and $\mathrm{C} 9$ which were difficult to discriminate during the early part of the growing season. The RGB image and the ground-truth reference are presented in Figure 1.

\subsection{Nonlinear subspace feature extraction}

To better understand nonlinear characteristics of hyperspectral data in the manifold domain. 3-D plots of selected coordinates for local ML are shown in Figure 2 for a tile, which contains 9 classes in the upper left corner of the original hyperspectral images. Figure 2 shows that nonlinear structures are clearly exhibited, Data structures provided by LE are significantly different from those of LLE and LTSA.LE also preserves the local neighborhood structures of the data, but not well preserves distance formation between the data.

For each of these nine tiles, manifold coordinates are derived using local ML methods. so, subspace feature images for each tile are obtained. The first three subspace feature images for all tiles are illustrated in Figure 3, we find that each feature image has different information; they clearly reflect different gray value for the different classes. Image texture is more abundant. The information of the first three feature images does not change obviously. Because local ML methods retain local structure of the data, the discontinuities of adjoining feature images are smaller.
In this study, we assumed that manifold coordinates of each tile can be thought to lie on a unified manifold coordinate system.In order to further evaluate the effectiveness of the full-scene global manifold coordinate system. Two DR results derived from single-tile manifold coordinate system and full-scene global manifold coordinate system respectively in combination with SVM-based classification are compared. Two ways of DR methods were utilized (1)Each local ML runs directly on the original all train samples and randomly sampled $10 \%$ of original all test data. (2) Each ML runs directly on the original all train samples, DR data of randomly sampled $10 \%$ of original all test data are obtained from full-scene global manifold coordinate system. SVM classifier was then used to classify DR data obtained by above two ways of ML methods with 20 replications of each experiment. Classification results are shown in Figure 4. For each ML,they have very similar classification results. Through the quantitative evaluation of classification accuracy, it further indicates the full-scene global manifold coordinate system of each local ML is effective.

\subsection{Comparison of pixel-based and object-based subspace feature classification}

Full-scene manifold coordinates are derived with local ML, meanwhile, subspace feature images are obtained with full-scene manifold coordinates for each ML. Subspace feature images with the use of SVM classifier are investigated.The pixel-based classification accuracies are shown in Figure 5 for each subspace feature images. The overall accuracies (OA) based on the confusion matrix; over a range of the parameter values for local ML methods are used to assess the classification results.

The statistics in Figure 5 are obtained using a pixel-by-pixel SVM classification without considering the spatial relationship of neighboring pixels. In Fgure 5, the $x$ axe represented the dimensionality of subspace, in general, we hope that ML methods are better able to capture the nonlinear structure of the associated manifolds and achieve higher accuracies with small 


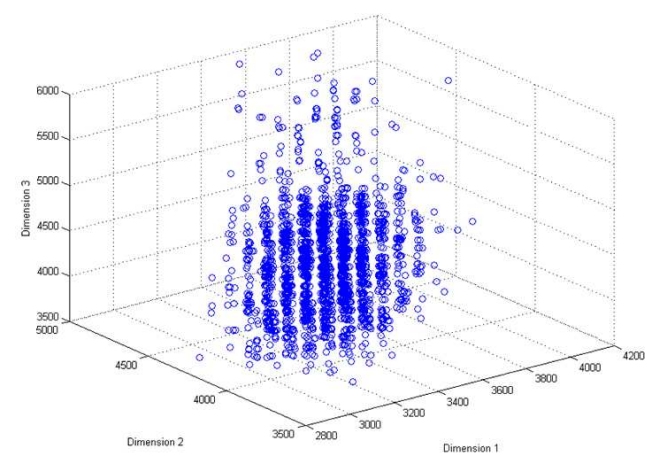

(a)

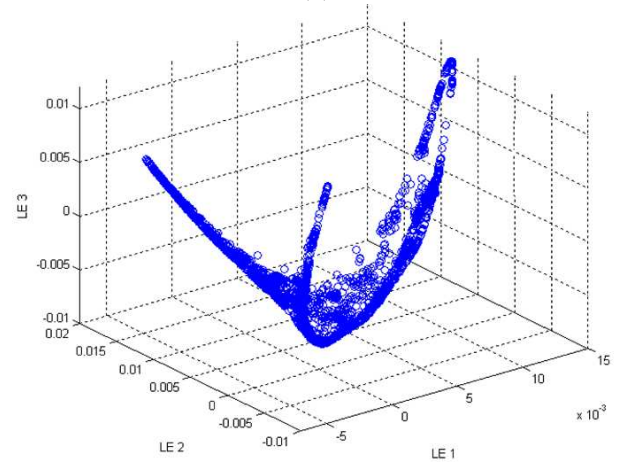

(c)

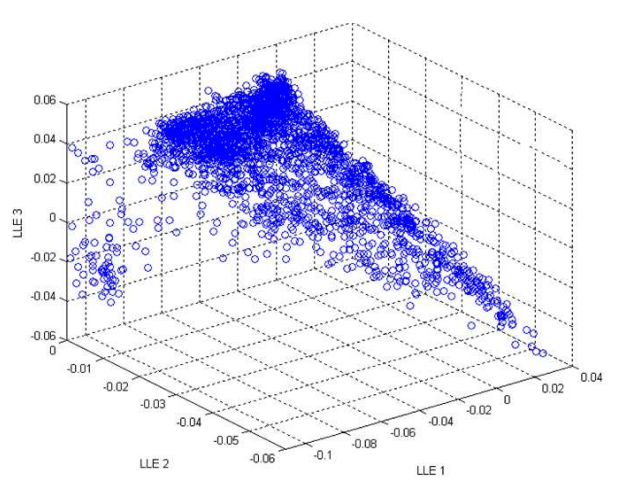

(b)

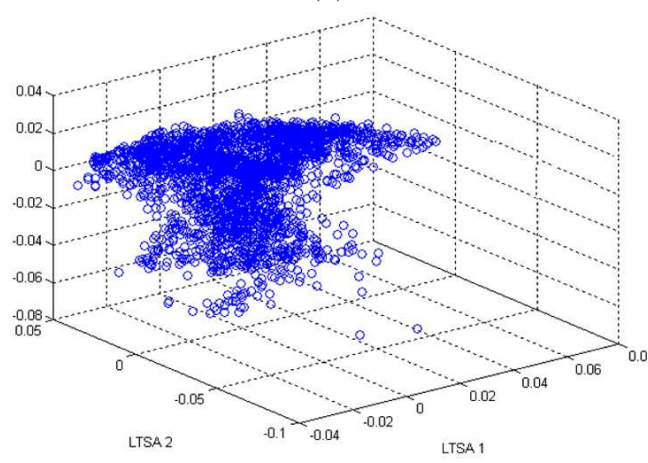

(d)

Fig. 2: 3D plots of the first three coordinates for (a) original high-dimensional space (b) LLE DR results (c) LE DR results (d) LTSA DR results

number of dimension. In Figure 5(a) to Figure 5(c), good performance can be achieved for more than 15 dimension with large values of . For smaller numbers of local neighbors $k$,larger values of dimension are required to achieve higher accuracies. In general, smaller numbers of local neighbors $k$ can capture the smaller neighborhood structure of data, so, it requires more bands to describle more details of local structure of the data, but larger numbers of local neighbors $k$ can smooth some of the details of local structure of the data, but it can better able to take into account the data global structure, and a small amount of band can better describe the data structure information. When implemented with best parameters, LLE and LTSA consistently yield better results than LE. Considering that the pixel-wise classification of SVM with the originally 200-dimensional AVIRIS channels gives $\mathrm{OA}=72.8 \%$, it can be said that subspace analysis is effective in extracting spectral information from the hyperspectral data,furthermore, it is able to reduce the computational cost.

Table 2 shows the object-oriented classification accuracies with scale/optimal segment size of each local ML method considering their best performance in pixel-based classification accuracies and The segmentation parameters are mainly based on experience by repeating tests and comparison with class label maps.
Table 2 shows that the object-oriented subspace feature classification can provide substantially higher accuracies than the pixel-wise classification. With the OSFA classification, the OA improvements are $12.7 \%, 8.7 \%$, $8.3 \%$ and $10.2 \%$ for 15 -dimemsional LLE, LTSA and LE features, respectively. The OSFA-based LLE, LTSA, LE features give over $90 \%$ overall accuracies. Therefore, it can be stated that the prosed OSFA can effectively exploit the spatial relationship of pixels and give much more accurate classification results.1

Figure 6 compares the classification maps of the LLE, LTSA and LE features for the pixel-based and object-based classification considering best performance in the classification accuracies. From the Figure 6, it can be observed that the OSFA-based method reduces the "pepperCsalt" effects resulted from the pixel-wise classification, and it avoids the misclassifications and uncertainty in homogeneous regions. In addition, the OSFA method classifies the image based on objects; consequently, it is more appropriate for the vector-based post-processing and Geographic Information System spatial analysis. Table 3 provides the class-specific accuracies for different subspace features with and without object-oriented analysis, and the accuracies obtained by all-bands AVIRIS hyperspectral data (200 channels) are used for comparison.it indicates that $\mathrm{C} 2$ and 


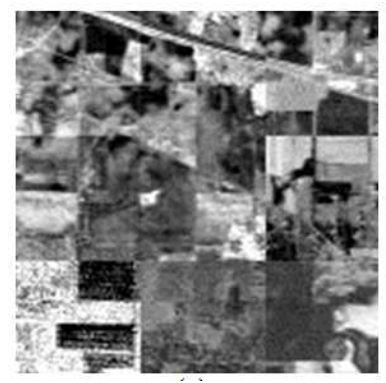

(a)

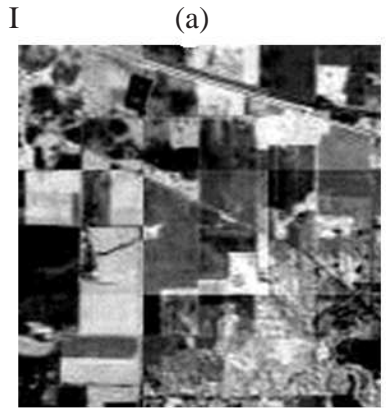

(a)

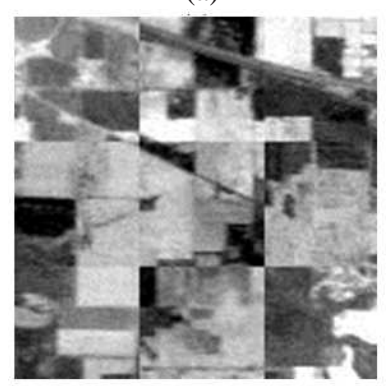

III

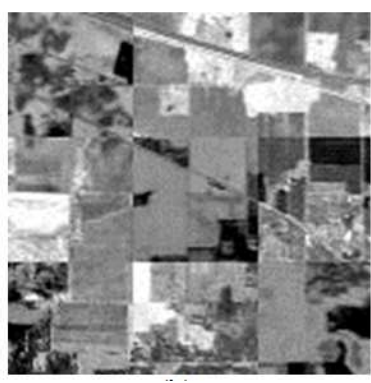

(b)

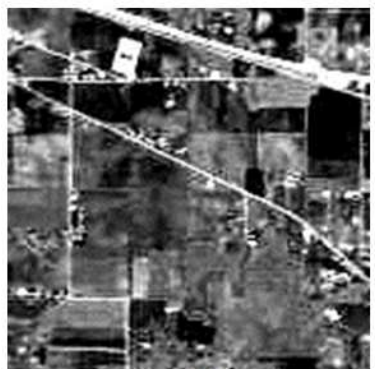

(b)

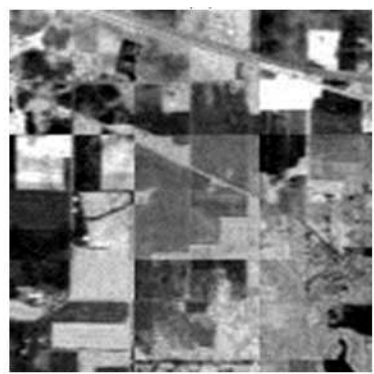

(b)

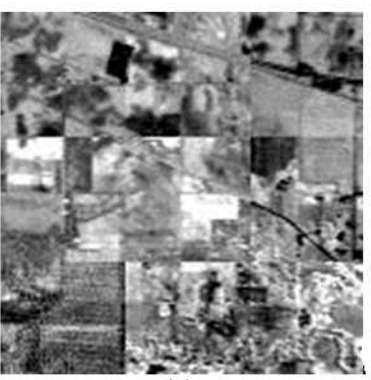

(c)

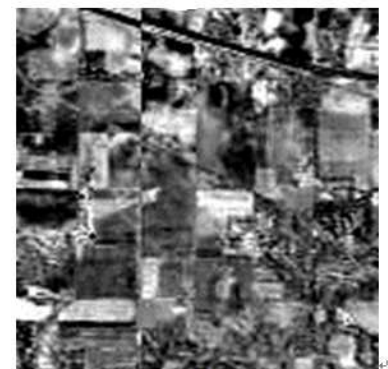

(c)

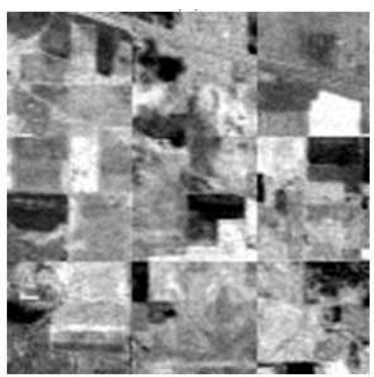

(c)

Fig. 3: The first three manifold coordinates derived for each tile using (I) LLE (II) LTSA (III) LE, from (a) to (c): coordinates 1, 2, 3

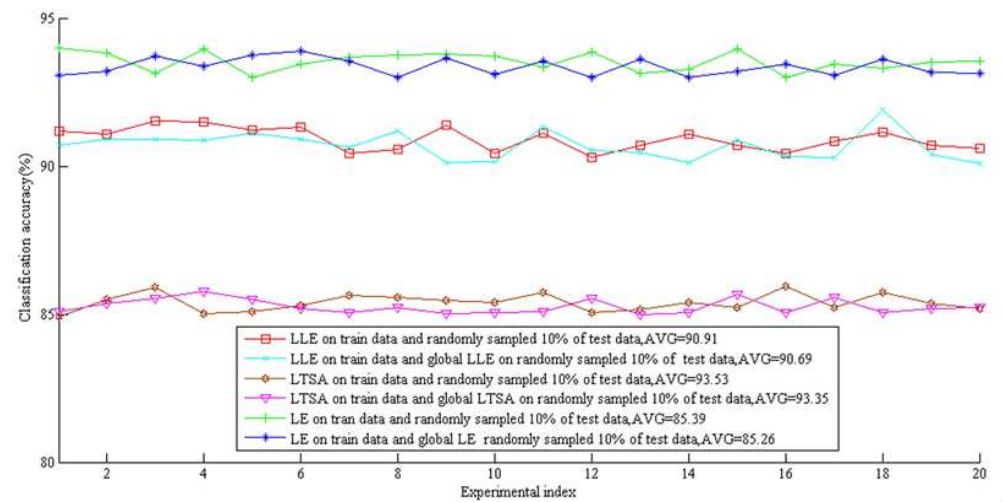

Fig. 4: Overall accuracies in percentage for two DR results from (1) ML on training data and test data, (2)ML on training data and full-scene global ML on test data, the average(AVG) of each classification accuracy are shown. 

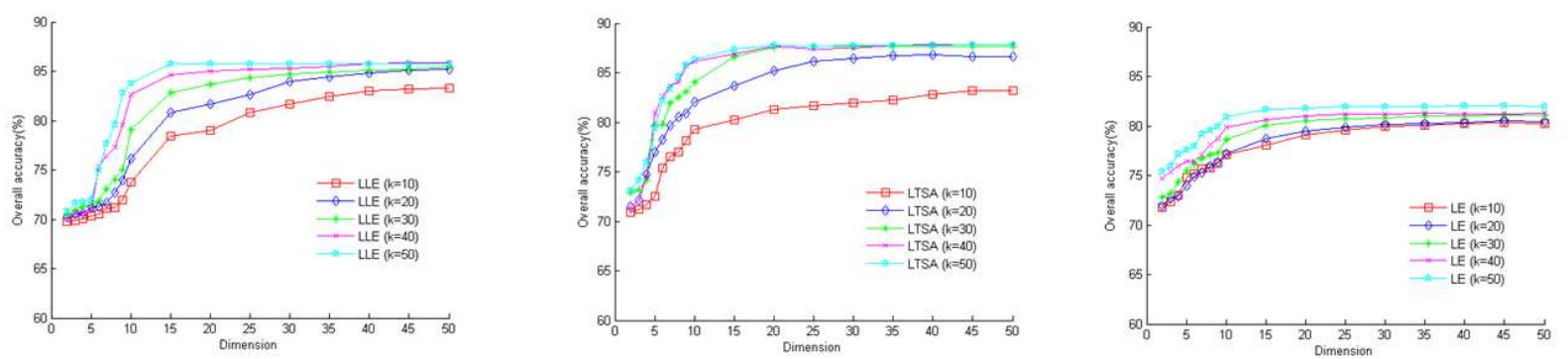

Fig. 5: Overall accuracies in percentage for the pixel-based classification for LLE, LTSA, LE

Table 2: Scale/optimal segment size and object-based classification accuracies with best parameter values of ML method ( $\mathrm{S}=$ shape, $\mathrm{O}=$ compactness).

\begin{tabular}{cccc}
\hline Name & LLE & LTSA & LE \\
\hline Parameter values & $k=50, p=20$ & $k=50, p=20$ & $k=50, p=20$ \\
Scale/optimal segment size & $10 / S_{0.7} O_{0.5}$ & $10 / S_{0.6} O_{0.4}$ & $5 / S_{0.6} O_{0.3}$ \\
Pixel-based OA & $85.7 \%$ & $87.0 \%$ & $81.9 \%$ \\
OSFA-based OA & $94.4 \%$ & $95.3 \%$ & $92.1 \%$ \\
\hline
\end{tabular}

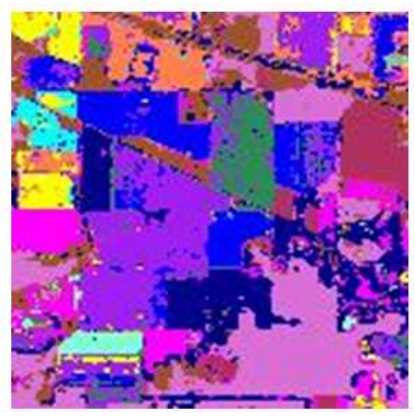

(a)

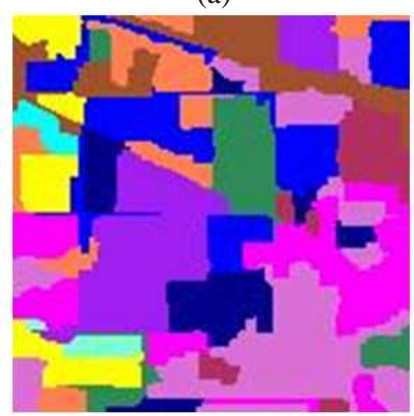

(d)

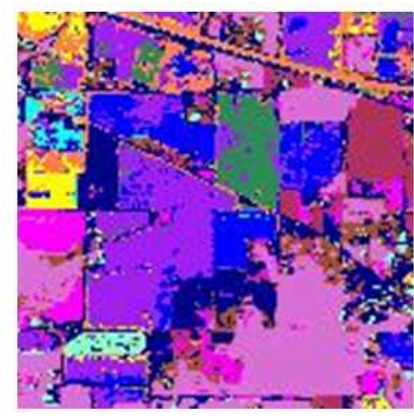

(b)

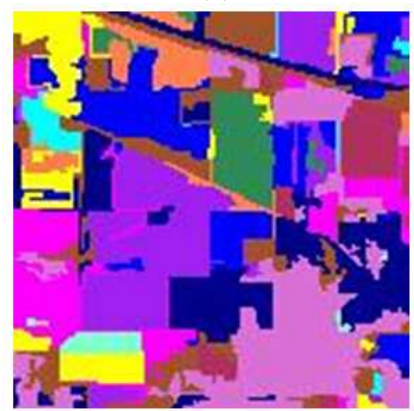

(e)

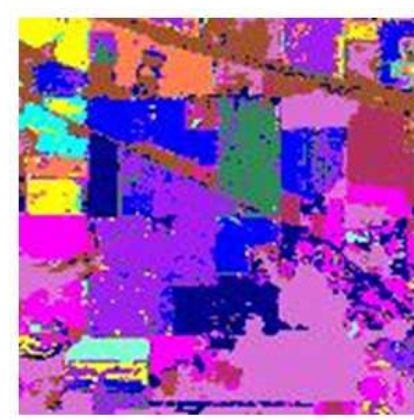

(c)

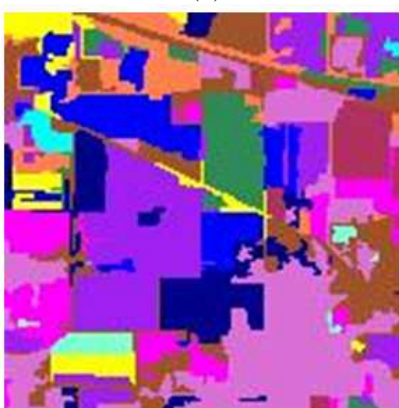

(f)

Fig. 6: Pixel-based classification maps for (a) LLE, (b) LTSA,(c)LE,object-oriented classification for (d)LLE, (e) LTSA,(f) LE.

C9, the most difficult class pair, can be well separated by ML features.

Textural measures have been proven an effective approach for spatial information extraction. Therefore, in order to further validate the proposed OSFA method. Grey-level co-occurrence matrix (GLCM) [27] and Gabor wavelet [28] were implemented as benchmarks. 20-dimensional LLE, LTSA, LE features were selected for the texture analysis considering good performance.

The accuracy improvements resulted from the GLCM textures were $5.2 \%, 3.4 \%$ and $7.8 \%$ for LLE,LTSA and LE, respectively, and the respective improvements from the wavelet texture were $2.7 \%, 2.2 \%$ and $3.6 \%$.However, it could be clearly seen that the OSFA-based classification obtained the best accurate results. However, the utility of 
Table 3: Class-specific accuracies in percentage from pixel-based $(\mathrm{P})$ and object-oriented $(\mathrm{O})$ classification for nonlinear subspace features

\begin{tabular}{cccccccc}
\hline \multirow{2}{*}{ Name } & \multirow{2}{*}{ 200 bands } & \multicolumn{2}{c}{ LLE } & \multicolumn{2}{c}{ LTSA } & \multicolumn{2}{c}{ LE } \\
\cline { 3 - 7 } & & $\mathrm{P}$ & $\mathrm{O}$ & $\mathrm{P}$ & $\mathrm{O}$ & $\mathrm{P}$ & $\mathrm{O}$ \\
\hline C1 & 60.7 & 79.8 & 90.1 & 80.6 & 95.6 & 80.3 & 89.6 \\
C2 & 56.4 & 89.7 & 93.9 & 90.4 & 95.1 & 87.6 & 92.6 \\
C3 & 75.2 & 71.4 & 90.1 & 89.8 & 90.9 & 70.1 & 93.3 \\
C4 & 85.1 & 91.2 & 95.7 & 95.3 & 97.1 & 87.7 & 91.7 \\
C5 & 72.6 & 88.7 & 96.9 & 90.2 & 94.9 & 81.4 & 96.2 \\
C6 & 79.4 & 86.4 & 98.0 & 97.6 & 98.6 & 84.2 & 89.6 \\
C7 & 61.7 & 78.3 & 95.7 & 87.7 & 96.6 & 78.9 & 95.2 \\
C8 & 84.1 & 86.8 & 93.5 & 85.9 & 94.6 & 81.9 & 88.9 \\
C9 & 68.4 & 87.6 & 95.9 & 83.6 & 95.1 & 88.3 & 95.6 \\
C10 & 87.8 & 89.2 & 97.8 & 80.1 & 96.2 & 87.6 & 89.5 \\
C11 & 79.5 & 91.8 & 95.6 & 87.9 & 94.3 & 78.2 & 94.5 \\
C12 & 60.1 & 78.6 & 96.5 & 82.8 & 97.2 & 80.0 & 96.1 \\
\hline
\end{tabular}

GLCM and wavelet textures substantially increases the number of dimensionality of features. Therefore, it can be said that the proposed OSFA-based classification is more effective in simultaneously exploit spectral and spatial information in terms of accuracies and computational cost.

\section{Conclusions}

In this study, a new object-oriented nonlinear subspace feature analysis has been presented to improve the performance of hyperspectral remote sensing images classification, in order to exploit the spectral and spatial information contained in the images. The nonlinear subspace characteristics of hyperspectral data are investigated using local ML,since local ML methods are able to model nonlinear structure in hypespectral data and reduce the information redundancy in hyperspectral data with hundreds of highly correlated bands. The local ML methods are utilized to obtain low dimensional feature images of hyperspectral imagery. Extracted features are implemented in conjunction with SVM classifier for pixel-based and object-oriented classification.

The experiments show that the object-oriented features analysis gives much more accurate mapping results than the pixel-wise classification. All nonlinear subspace features give higher classification accuracies than the full dimensional hyperspectral images. It can be said that the integration of nonlinrear subspace analysis and object-based processing is effective for spectral/spatial information extraction and classification from hyperspectral data. In addition, in comparison with results obtained by GLCM and Gabor wavelet textures classification, the proposed approach gives obviously higher accuracies but with much smaller feature dimensionality, with a proper selection of parameters and a sufficient number of features, LLE and LTSA consistently yield better results than LE.

\section{Acknowledgement}

This work was supported by the National High Technology Research and Development Program of China under Grant 2012AA12A304.

\section{References}

[1] M. A. Karaska, R. L. Hugenin, M. Wang, J. R. Jenson, R. S. Kaufmann, Photogrammetric Engineering and Remote Sensing, 70, 125-133 (2004).

[2] G. Hughes, IEEE Transactions on Information Theory, 14, 55-63 (1968).

[3] S. Tadjudin, D. A.Landgrebe, IEEE Transactions on Geoscience and Remote Sensing, 37, 2113-2118 (1999).

[4] M. Chi, L.Bruzzione, IEEE Transactions on Geoscience and Remote Sensing, 45, 1870-1880 (2007).

[5] C. M. Bachmann, T. L.Ainsworth, R. A. Fushina, IEEE Transactions on Geoscience and Remote Sensing, 43, 441454 (2005).

[6] R. Saegusa, H. Sakano, S.Hashimoto, Neurocomputing, 61, 57-70 (2004).

[7] A. A. Green, M. Berman, P. Switzer, M. D. Craig,IEEE Transactions on Geoscience and Remote Sensing, 26, 65-74 (1988).

[8] B. Kuo, D. A. Landgrebe, IEEE Transactions on Geoscience and Remote Sensing, 42, 1096-1105 (2004).

[9] M. Pal, and G. M. Foody, IEEE Transactions on Geoscience Remote Sensing, 48, 2297-2307 (2010).

[10] C. M.Bachmann, T. L.Ainsworth, R. A. Fusina, M. J.Montes, J. H.Bowles, D. R.Korwan, and D. B.Gillis, IEEE Transactions on Geoscience Remote Sensing, 47, 884-897 (2009).

[11] J. B.Tenenbaum, V. de Silva, J. C.Langford, Science, 290, 2319-2323 (2000).

[12] B. Schölkopf, A. J. Smola, K. R. Muller, Lecture Notes in Computer Science, 1327, 583-588 (1997).

[13] S. T. Roweis, L. K Saul, Science, 290, 2323-2326 (2000).

[14] Z. Y. Zhang, H. Y. Zha, SIAM J. Scientific Computing, 26, 313-338 (2004). 
[15] M. Belkin, P. Niy ö gi, Neural Computation, 15, 1373-1396 (2003).

[16] M. Fauvel, J. Chanussot, J. A Benediktssion, EURASIP Journal on Advances in Signal Processing, 783194 (2009).

[17] X. Li, S. Lin, S. Yan, D. Xu, IEEE Transactions on Systems, Man, and Cybernetics, Part B, Cybernetics, 38, 342352 (2009).

[18] L. Ma, M. M.Crawford, J. W. Tian, IEEE Transactions on Geocience Remote Sensing, 48, 4099-4199 (2010).

[19] J. M. Duarte-Carvajalino, G. Sapiro, M. Velez-Reyes, P. E. Castillo, IEEE Transactions on Geoscience and Remote Sensing, 46, 2418-2434 (2008).

[20] X. Huang, L. Zhang, International Journal of Remote Sensing, 30, 3205-3221 (2009).

[21] Definiens, Definiens Developer 7.0 Reference Book. Definiens AG, München, Germany, (2007).

[22] R. Mathieu, J. Aryal, Planning and Urban Ecology, 81, 179192 (2007).

[23] VL. Lucieer, International Journal of Remote Sensing, 29, 905-921(2008).

[24] U. C. Benz, P. Hofmann,G. Willhauck, I. Lingenfelder, M. Heynen, ISPRS Journal of Photogrammetry and Remote Sensing, 58, 239-258 (2004).

[25] B. A. Johnson, Remote Sensing Letters, 4, 131-140 (2013).

[26] X. J. Li, Q. Y. Meng, X. F. Gu, T.Jancso, T. Yu, K.Wang, S.Mavromatis, International Journal of Remote Sensing, 34, 4655-4668 (2013).

[27] B. S. Manjunath, W. Y. Ma, IEEE Transactions on Pattern Analysis and Machine Intelligence, 18, 837-842 (1996).

[28] Y. O. Ouma, T. G. Ngigi, R. Tateishi, International Journal of Remote Sensing, 27, 73-104 (2006).

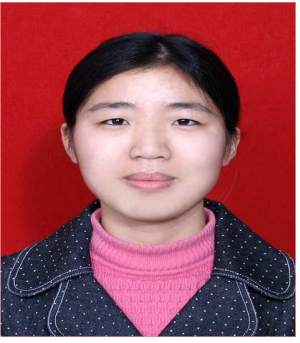

Ling Ding received the B.E. and the M.E. degree both from the Shandong University of Science and Technology. She is currently pursuing the Ph.D. degree at the Institute of Remote Sensing and Digital Earth, Chinese Academy of Sciences. Her research interests include machine learning, neural networks, and algorithms analysis with applications to identification and classification processes for remote sensing data.

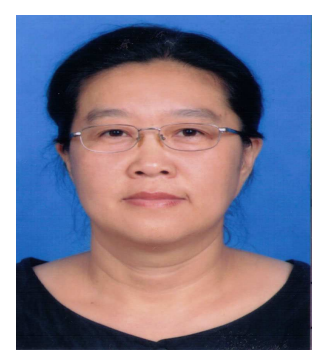

Ping Tang received the B.S. degree in mathematics from Ningxia University, and the M.S. and Ph.D. degrees both in Mathematics from Beijing Normal University. She is a Professor in Institute of Remote Sensing and Digital Earth, Chinese Academy of Sciences. Her research lies in using mathematical theories to develop algorithms for satellite image processing such as multispectral imagery radiometric and geometric correction.

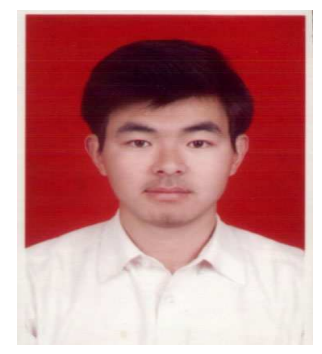

Hongyi Li received the B.E. and the M.E. degree both from Ningxia University.Presently he serves as assistant researcher in Institute of Remote Sensing and Digital Earth, Chinese Academy of Sciences.His research lies in images processing and analysis such as designing software systems for satellite image processing and applications,data mining and scientific programming. 